\section{Psicologia Escolar \\ e Educacional}

ARTí́CULO

DOI: http://dx.doi.org/10.1590/2175-35392021221423

Localizador - e221423

\title{
TEORÍAS SUBJETIVAS DE LA CONVIVENCIA ESCOLAR: ¿QUÉ DICEN LOS PADRES?
}

\author{
David Cuadra-Martínez ${ }^{1} \mathbb{D}$ Pablo J. Castro-Carrasco ${ }^{2 ; 3} \mathbb{D}$; Luciene Regina Paulino Tognetta ${ }^{4} \mathbb{D}$; Daniel Pérez- \\ Zapata $^{3,5} \mathbb{D}$; Douglas Véliz-Vergara ${ }^{1} \mathbb{D}$; Néstor Menares-Ossandón ${ }^{1} \mathbb{D}$
}

\section{RESUMEN}

El objetivo de este trabajo fue describir e interpretar las TS de la convivencia escolar, de un grupo de padres y madres de una escuela de educación primaria chilena. Se utilizó un muestreo teórico y se aplicaron grupos de discusión y entrevistas episódicas a 16 padres y madres de una escuela de educación primaria. Los datos se analizaron mediante la técnica de la teoría fundamentada, encontrando (a) teorías subjetivas simples, pero que disponen a los padres a educar a sus hijos en convivencia; (b) factores asociados a la CE y propuestas para su mejora, (c) además de un rol parental educativo que se organiza en tres niveles para enseñar a sus hijos a convivir en la escuela. En la discusión se analiza la implicancia de estas teorías subjetivas en la convivencia escolar y el rol parental.

Palabras clave: convivencia escolar; teorías subjetivas; rol parental.

\section{Subjective theories about school coexistence: What do parent say?}

\begin{abstract}
The aim of this work was to describe and to interpret subjective theories about school coexistence in a group of parents from a Chilean primary school. A theoretical sample was used, group discussions and episodic interviews were conducted to 16 parents from a primary school. Data was analyzed thorough grounded theory, finding (a) simple subjective theories lead parents to raise their children on an environment-based approach; (b) associated factors with schooling environment and emerging proposals to improve them; (c) as well as a schooling parental role that it organizes around three levels of climate schooling teaching. In the discussion section, we analyze implications of these subjective theories into schooling environment and parental role.
\end{abstract}

Keywords: school coexistence; subjective theories; parental role.

\section{Teorias subjetivas da convivência escolar: o que os pais dizem?}

\section{RESUMO}

O objetivo deste trabalho foi descrever e interpretar as teorias subjetivas (TS) da convivência escolar (CE), de um grupo de pais e mães de uma escola de educação primária chilena. Uma amostragem teórica foi utilizada e se aplicaram grupos de discussão e entrevistas episódicas em 16 pais e mães de uma escola de educação primária. Os dados foram analisados através da técnica da teoria fundamentada (Grounded Theory), encontrando: (a) teorias subjetivas simples, mas que proporcionam aos pais educar seus filhos na convivência; (b) fatores associados à CE e propostas de melhoria; (c) além de um papel educativo dos pais que se organiza em três níveis para ensinar seus filhos a conviver na escola. Na discussão é analisada a implicação das teorias subjetivas na convivência escolar e no papel parental.

Palavras-chave: Convivência escolar; teorias subjetivas; papel dos pais.

\footnotetext{
1 Universidad de Atacama - Copiapó - Chile; david.cuadra@uda.cl; douglas.veliz@uda.cl; nestor.menares@uda.cl

2 Universidad de La Serena - Coquimbo - Chile; pablocastro@userena.cl

${ }^{3}$ Universidad Católica del Maule - Talca - Chile; pablocastro@userena.cl; DIP827@student.bham.ac.uk

${ }^{4}$ Universidade Estadual Paulista Júlio de Mesquita Filho - Araraquara, SP - Brasil; Irpaulino@uol.com.br

${ }^{5}$ University of Birmingham, School of Psychology - Birmingham - United Kingdom; DIP827@student.bham.ac.uk
} 


\section{INTRODUCIÓN}

La convivencia escolar (CE) es un tema importante dentro de las políticas educativas a nivel internacional (Ascorra, López, Carrasco, Pizarro, Cuadros, \& Núñez, 2018). Los resultados de diferentes investigaciones (Román \& Murillo, 2011) señalan que la calidad de las relaciones interpersonales de la escuela afecta directamente el desempeño de los estudiantes, denotando así la importancia de generar espacios en que el bienestar esté presente en la escuela. Incluso, las temáticas de la convivencia, resolución de conflictos, cooperación y trabajo en equipo, son reconocidas como elementos de evaluaciones internacionales dada su importancia en el escenario educativo (OCDE, 2013).

Autores como Del Rey, Ortega y Feria (2009) definen CE como un principio fundamental para una educación participativa, democrática, ciudadana y bien integrada curricularmente, que se gesta a través de la vida en común en todos los espacios escolares. Esto, mediante un proceso permanente de aprendizaje de la competencia social y afectiva para establecer vínculos interpersonales centrados en la democracia, el respeto, la empatía, la tolerancia y la solidaridad.

Respecto a la familia, se asume que la relación que establece con la escuela es fundamental para la adaptación del estudiante y el logro académico (Shute, Hansen, Underwood, \& Razzouk, 2011). La funcionalidad familiar incide de manera importante en la CE, dado que puede actuar como un factor protector de las relaciones interpersonales (López et al., 2013), o un factor de riesgo para la generación de un clima escolar negativo (Cid et al., 2008; Senra, Lourenço, \& Pereira, 2011). Incluso, las investigaciones muestran que los educadores consideran a la familia como única causa o de mayor influencia en los comportamientos de los estudiantes en la escuela (Vinha \& Tognetta, 2013).

No obstante lo anterior, hay evidencias de una crisis en la participación de los padres en la escuela, sobre todo de aquellos grupos con alta vulnerabilidad social (Acuña, 2016). De esta manera, son frecuentes las propuestas para el trabajo con padres y madres que se centran en fomentar su participación en la toma de decisiones educativas (Alcalay, Milicic, \& Torretti, 2005; Sandoval, 2014), la mejora de la comunicación con la escuela (Acuña, 2016) y la educación parental (Tognetta \& De Nadai, 2018).

Lograr una alianza familia escuela para el aprendizaje de la $C E$, requiere considerar las vivencias y significados construidos por cada uno de los actores educativos en las comunidades escolares (Ascorra et al., 2018). No obstante, la mayoría de los estudios en la temática incorporan mucho más los significados de los docentes, otros profesionales y estudiantes, considerando menos los de padres y madres (Ascorra et al., 2018; Saldivia, 2008).

Cuando los hijos(as) ingresan a la escuela, los padres elaboran expectativas y creencias sobre el éxito académico, la adaptación del estudiante e incluso el futuro profesional (Rahmawati, Tairas, \& Nawangsari, 2018). No obstante, éstos le otorgan más importancia a la preparación académica del estudiante, que a las habilidades sociales para convivir en la escuela (Kaufman, 2009). Cuando se describen las creencias de los padres acerca de las habilidades socioemocionales de los estudiantes que son relevantes para la funcionalidad en la escuela, estos refieren que la capacidad para interactuar con sus pares y de plantear su opinión en clases son importantes (Rahmawati et al., 2018). Estudios latinoamericanos evidencian que aunque los padres se encuentran preocupados por la violencia escolar, tienden a minimizarla al interior de su escuela (Fondo de las Naciones Unidas para la Infancia, 2011). También que los problemas de convivencia se deben a su baja participación e interés en la educación de sus hijos (López et al., 2011; Saldivia, 2008; Vinha \& Tognetta, 2009) o a la poca valoración que la escuela hace de ellos (Griffith, 2001). Resultados similares se han encontrado en escuelas con vulnerabilidad social, en donde las relaciones familia escuela se caracterizan por la desconfianza y el fracaso es interpretado por los padres que ejercen liderazgo en las escuelas, como una responsabilidad familiar (Montecinos, Sisto, \& Ahumada, 2010).

En este estudio, se ha optado por indagar la CE desde las teorías subjetivas (TS) de los padres. Las TS son un tipo de representación cognitiva individual y/o colectiva de mayor elaboración, complejidad y generalidad, que permite explicar, organizar y modular la acción, definir situaciones y justificar el propio comportamiento (Catalán, 2010; Flick, 2007). El objetivo general de este trabajo es describir e interpretar las TS de la CE, de un grupo de padres y madres de una escuela de educación primaria chilena. A nivel específico se buscó: (a) describir el significado subjetivo de CE y la forma en que los participantes caracterizan la convivencia de la escuela, (b) describir las explicaciones que presentan de la $\mathrm{CE}$, (c) determinar cómo explican el rol parental educativo en la CE y (d) proponer un modelo comprensivo de la $\mathrm{CE}$, en función de las TS encontradas.

\section{MÉTODO}

Corresponde a un estudio descriptivo interpretativo que utilizó metodología cualitativa (Taylor \& Bogdan, 1987), puesto que se buscó caracterizar la CE desde el marco interpretativo de los padres y madres. El diseño fue de estudio de caso (Stake, 1999). 


\section{Muestra}

Se realizó un muestro teórico, buscando la representatividad de casos, incluyendo a: (a) padres o madres que forman parte de la directiva del subcentros y quienes no la constituyen; (b) padres o madres de estudiantes con alto y bajo rendimiento académico y (c) padres o madres de estudiantes con y sin problemas disciplinarios en el aula. Se trabajó con 16 padres y madres del primer ciclo de educación primaria de una escuela pública de la región de Atacama, Chile: 6 padres y madres de las directivas de los subcentros ${ }^{1}$ de los cursos primero, tercero y cuarto año básico y 10 del tercer año básico que no constituyeron la directiva del subcentro. Un $94 \%$ fueron mujeres (madre) y un $6 \%$ hombres (padre). El $81 \%$ de ellos tiene una edad de entre 25 y 40 años, mientras que el $19 \%$, mayor a 50 años. Además, el $19 \%$ califica en un nivel socioeconómico medio y el $81 \%$ en uno bajo. Las familias que integran la comunidad educativa presentan un nivel socioeconómico medio bajo y entre un $62 \%$ y $80 \%$ de vulnerabilidad social, según la evaluación que realiza el Ministerio de Educación de Chile.

\section{Procedimiento de recolección de información}

Se contactó a la dirección de escuela y a los padres y madres, a quienes se les explicó los objetivos y metodología de la investigación, además de los criterios éticos. Quienes aceptaron participar del estudio firmaron un consentimiento informado. En primer lugar se realizaron dos grupos de discusión, cada uno constituido por cinco madres y/o padres del tercer año básico. Su implementación se basó en un guión temático que consideró como ejes indagatorios: (a) significado de CE, (b) características de la $\mathrm{CE}$, (c) explicaciones acerca de la $\mathrm{CE}$, (d) rol parental educativo en la CE y (e) propuestas para mejorar la CE. Cada grupo de discusión tuvo una duración aproximada de 1 hora y los datos se grabaron en audio. Posteriormente se aplicaron entrevistas episódicas, instrumento que posibilitó profundizar en las teorías subjetivas colectivas encontradas mediante el primer instrumento y contrastar estos hallazgos preliminares, a fin de otorgarle mayor credibilidad al estudio. La entrevista episódica es un instrumento de recolección de información especialmente indicado para reconstruir teorías subjetivas en base a episodios biográficos (Flick, 2007). Se aplicaron 6 entrevistas episódicas a 6 madres con cargos directivos del subcentro: 2 de primer año básico, 2 de tercer año básico y 2 de cuarto año básico,

\footnotetext{
${ }^{1}$ En Chile los padres y madres de una escuela se organizan jurídicamente en Centros de Padres y Apoderados. Este último término se utiliza para denotar a quien asume la responsabilidad educativa del hijo y se vincula con la escuela. El Subcentro corresponde a los apoderados de un curso de la escuela, organizados mediante una directiva.
}

utilizando el mismo guión temático de los grupos de discusión. La entrevista episódica tuvo una duración aproximada de 1 hora y sus datos se grabaron en audio.

\section{Procedimiento de análisis de datos}

Las transcripciones de los grupos de discusión se sometieron a análisis de contenido según la técnica de la teoría fundamentada (Strauss \& Corbin, 2002). El análisis simultáneo de los datos obtenidos mediante los grupos de discusión permitió guiar la indagación mediante las entrevistas episódicas, información que posteriormente fue analizada mediante la misma técnica señalada. De esta manera, se realizaron tres tipos de codificaciones: (a) codificación abierta, en donde mediante análisis comparativo constante, se identificó en el texto transcrito frases, oraciones y/o párrafos que contuvieran enunciados explicativos explícitos, tomando como referencia las expresiones (Flick, 2007) relacionados con el problema de estudio y también implícitos, inferidos por el investigador, transformándolos en códigos que permitieron acotar la información; (b) codificación axial, en donde los códigos elaborados se sometieron a análisis comparativo constante a fin de construir categorías representativas del problema de estudio y (c) codificación selectiva, que permitió la construcción de un modelo comprensivo de las TS encontradas, estableciendo relaciones entre las categorías y asignando a una de éstas como núcleo de este proceso. En este trabajo, siguiendo la sugerencia de Flick (2007) se presenta el resultado de la codificación selectiva mediante un breve texto explicativo y una figura.

\section{RESULTADOS}

\section{Significado subjetivo de convivencia escolar}

Los padres y madres entienden la CE como una relación interpersonal positiva entre los/las estudiantes y entre los/las estudiantes y los docentes, que se manifiesta dentro y fuera del aula de clases. Los indicadores que dan cuenta de la CE son: (a) el compartir entre estudiantes, (b) el respeto en la relación profesor alumno y entre los estudiantes y (c) el buen trato entre estos actores educativos.

$$
\begin{aligned}
& \text { Llevarse bien con los compañeros, compartir. } \\
& \left(\mathrm{p} 2, \mathrm{gd}^{2}\right. \text { ) } \\
& \text { El buen trato, el llevarse bien todo el conjunto de } \\
& \text { niños que se lleven bien, que jueguen, no pelear, } \\
& \text { para mí esa es la convivencia. (e1). }
\end{aligned}
$$

Los participantes explican que un cierto grado de desobediencia e indisciplina en los estudiantes, son

\footnotetext{
$\overline{{ }^{2} \mathrm{P}=\text { Participante; }} \mathrm{gd}=$ grupo de discusión; e = entrevista
} 
elementos constitutivos de la $\mathrm{CE}$, considerándolos comportamientos normales en la interacción de los alumnos en la escuela. Esto probablemente implica un rol parental educativo que se ejerce con una relativa flexibilidad en la enseñanza de las normas y reglas para la $C E$, que el hijo/a debe implementar cuando integra grupos de pares:

Quizás en el colegio sí se vuelven más revoltoso porque están con sus amigos, se ponen así y no hacen caso, pero yo creo que es normal. (p1, gd1).

La CE también es entendida como un proceso que se inicia en la preescolaridad de manera positiva y que se va deteriorando cuando el estudiante va cursando niveles superiores de escolaridad. Lo anterior es explicado en base a una dificultad de los padres y madres para adecuarse al desarrollo de los hijos/as y a la mayor protección que la escuela le entrega a los preescolares.

... cuando los niños están chiquititos en los cursos de pre kínder o kínder es otra realidad la que uno vive dentro... porque es como más unión... que de repente los niños más grandes andan a garabato limpio... y se tratan mal entre ellos... los niños van creciendo... no sé, van adquiriendo otro tipo de cosas. (e2)

\section{Convivencia escolar en una escuela de alta vulnerabilidad social}

En general, los padres y madres consideran que la escuela está dispuesta a mejorar la CE, pero no planifica ni implementa actividades sistemáticas que apunten a promoverla. En este contexto, explican que el profesor está fundamentalmente centrado en la enseñanza de los contenidos académicos:

Es que yo creo que la escuela nunca quiere cosas malas, siempre busca cosas buenas para los niños (p1, gd2).

No se motivan [a realizar actividades de convivencia] (p3, gd1).

Me parece que falta interés de la profesora para hacer eso (p5, gd2).

A nivel de apoderados, identifican un clima social escolar negativo, caracterizado por una baja participación en las actividades del curso y un alto nivel de conflictividad. Esta última situación también se presenta en los estudiantes.

La relación de convivencia entre profesor y alumno también es evaluada de manera negativa: consideran que es conflictiva, que el docente posee un bajo control para abordar la disciplina de los estudiantes y que estos últimos tienden a romper las normas. Explican que lo anterior conlleva a que el docente frecuentemente tenga que resolver los conflictos de manera reactiva y agresiva.

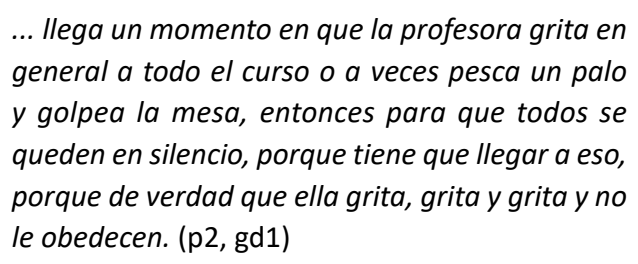

Como factores que obstaculizan la CE de su escuela, nombran (a) la falta de participación y comunicación entre los apoderados; (b) la insuficiente comunicación entre apoderados y docente; (c) la falta de asignación de tiempo y espacio por parte de la profesora, para educar a los estudiantes en CE y la limitada comunicación que tiene con sus estudiantes y (d) la insuficiente comunicación de los estudiantes con los padres y profesores cuando enfrentan conflictos.

Pero la profesora si... mi hijo hoy día se portó mal, ella sale y habla conmigo, pero si no hizo nada, no sale a hablar con uno, entonces uno no sabe. Cuando la ve: "ah, se portó mal", porque viene a decir algo, pero eso es lo único, cuando uno la ve. $(\mathrm{p} 5, \mathrm{gd} 2)$

\section{Factores asociados a la convivencia escolar}

La CE es explicada fundamentalmente en función del tipo de crianza que ejercen los padres y madres con sus hijos(as). Se asume que los valores y normas familiares transmitidas al hijo(a) son la base de la convivencia del estudiante.

Es que yo creo que eso viene de la base de la casa, de cómo es la convivencia en el colegio, entonces yo creo que todo parte de la casa, porque uno sabe cómo es en la casa y como que en el colegio va a ser igual. (p3, gd2)

La escuela también tiene un rol protagónico en la $\mathrm{CE}$, atribuyéndole el mismo impacto que la crianza familiar puede tener en ésta. Los participantes explican que el efecto que tiene la escuela en el aprendizaje de la CE de los estudiantes, se debe fundamentalmente a la gran cantidad de tiempo que éstos permanecen en este contexto. Para estos padres y madres, este último argumento representa un sustento del deber de la escuela de educar en convivencia.

Es que es cincuenta y cincuenta, cincuenta es en la casa y cincuenta por ciento es en el colegio, porque igual pasan la mayor parte del día en el colegio, entonces igual tienen que ver aquí cómo le enseñan. (p1, gd1)

Los participantes identifican un factor obstaculizador de su rol parental educativo y del de la escuela en el desarrollo y enseñanza de la CE. Consideran que la promulgación de los derechos de niño inhibe la corrección 
de las conductas indisciplinadas en los estudiantes(as), por parte de los profesores, los padres y madres, puesto que el castigo, que es considerado por ellos como una necesaria herramienta educativa, podría ser evaluado por la comunidad como una violación de estos derechos, situación que conllevaría a una sanción legal. De esta manera, para evitar consecuencias legales, explican que la tendencia es flexibilizar las normas de crianza y de la escuela. Desde esto, se hipotetiza una orientación a la acción que inhibe el rol parental educativo de la CE, a la hora de planificar e implementar medidas de regulación del comportamiento de sus hijos(as).

Entonces el alumno está como muy liberal en el establecimiento, por eso que están sucediendo muchas cosas, no solamente dentro de la sala sino que también en el interior de la escuela. Las normas, los profesores, porque los que estudiábamos antes en esta misma escuela no era así, entonces por eso le digo yo que por el asunto ese de los derechos del niño. ( $\mathrm{p} 2, \mathrm{gd} 2)$

Este grupo de padres y madres también identifica una serie de medidas que podrían mejorar la CE: (a) uso de tecnologías de la información y comunicación para fortalecer la comunicación entre el profesor y el apoderado, (b) la adecuación de la enseñanza, puesto que consideran que esto permitiría que todos los alumnos orienten su comportamiento hacia metas de aprendizaje; (c) la educación en valores y relaciones interpersonales y (d) el fortalecimiento del rol parental educativo.

... acudir a un especialista, porque yo lo hice y otra cosa es la comunicación y tratar con mucho cariño a nuestros hijos (e3).

Que la profesora dedique una media hora o veinte minutos a conversar, a dejar de hacer tarea y conversar con los niños: qué te parece tu compañero, tu compañera, entonces se van conociendo ellos mismos (p1, gd2).

\section{Rol parental educativo de la convivencia escolar}

En general, los padres y madres de los estudiantes consideran que educan para la $\mathrm{CE}$, aunque lo definen como un rol difícil de cumplir. Se observa a la base una teoría subjetiva que explica la enseñanza de la convivencia como un proceso que se debe implementar desde la primera infancia, etapa evolutiva que asumen como altamente significativa y determinante en el aprendizaje de la CE.

También, estos padres consideran a la violencia en la infancia como una característica propia de la sociedad actual, situación que podría dar cuenta de una naturalización de la misma.
Así lo percibo yo, los niños están muy violentos hoy en día, están demasiado violentos... (e6).

Yo creo que su caso es muy distinto, porque usted lo tiene desde ahora, usted no sabe cómo viene, porque tiene ocho años, entonces no sabe cómo viene de antes, quizás por eso usted lo tomó mal, ya está desordenado, se porta mal y usted lo está tratando de enderezar que es distinto. (p2, gd1)

Asimismo, los participantes utilizan un método de enseñanza de la CE que puede clasificarse como: (a) centrado en el diálogo, incorporando temas de conversación tales como el respeto, las normas y la manera de abordar los problemas de CE; (b) centrado en la manifestación de apoyo incondicional para resolver conflictos de CE y (c) el uso limitado del castigo, pese a ser considerado como una herramienta importante para la educación parental, dado que hay temor de verse involucrado en disputas legales al incumplir los derechos del niño(a).

... nosotros como familia, tratábamos de darle apoyo a ella no más ... que no hiciera caso, que ella era una niña habilosa, que no tenía que echarse a morir por cosas que no valían la pena a la larga [maltrato] ...porque era una persona que valía mucho. (e2)

Uno tiene que darse tiempo de conversar con él, decirle esto está mal, esto está bien. Tiene que tener modales, explicarle lo bueno y malo, si hiciste algo malo tienes que contarlo y lo vamos a conversar. El apoyo siempre, no importa que sea bueno o malo yo siempre lo voy a apoyar. (p2, gd1)

Como estrategia de educativa de la CE, los padres y madres incorporan la enseñanza de procedimientos para abordar conflictos interpersonales, esto es, patrones de acción que los hijos deben seguir sucesivamente frente a determinados conflictos escolares: (a) pedir al docente que medie en el conflicto, (b) solicitar autorización al profesor para salir del aula, a fin de buscar el apoyo de la inspectoría; (d) si el inspector no presta el apoyo necesario, informar del problema en el hogar, a fin de que finalmente sean los padres quienes medien para resolver el conflicto.

Si usted, un niño le dijo esto, le dijo esto otro, usted se para y le dice a la señorita: me dijo esto. Si la profesora no le escuchó a la primera, no le es escuchó a la segunda, no le escuchó a la tercera, usted pide permiso y se va a la inspectoría: pero es que no me deja, me avisa. (p4, gd2)

... tú vas donde la profesora, tú vas donde el inspector o tú vas donde la asistente o tú vas donde la tía, tú le dices el niño me golpeó, pero tú no tienes que golpear... a los 5 o 10 minutos volver a 
jugar, porque eres niño, porque tienes que pasar por esas etapas... (e6)

Finalmente, la pelea, entendida por ellos como una confrontación violenta entre pares, también es un procedimiento que algunos padres y madres enseñan a sus hijos a implementar cuando otras estrategias de resolución de conflictos no dan resultado. Así lo expresan algunos participantes:

No sé, mi hijo la mentalidad que tiene, lo que yo le digo: si me pegan... yo pego (p2, gd1).

... llegó el momento que él comenzó a hacer lo mismo [pelear]. Entonces qué hago yo como mamá, si yo le digo a él que no lo haga, las otras mamas ¿qué es lo que hacen?... hacen todo lo contrario... es decir, "no, tú tienes que hacerlo". (e4)

\section{Modelo comprensivo de la convivencia escolar, desde las TS de los padres}

Las TS de la CE de los padres, se organizan en base a significados y asunciones ontológicas y epistemológicas de lo que es la CE y cómo se enseña y aprende, incluyendo niveles más prácticos y concretos desde donde ejercen este rol educativo, que considera métodos y procedimientos. Estas TS se desarrollan en base a distintos saberes acerca de cómo criar y educar a los hijos, uno de estos es el que transmite la escuela, conocimiento que refleja falencias importantes en el abordaje de la CE. Esto produce confusiones en los padres o limitaciones en la forma en que enfrentan el rol de enseñar a convivir a sus hijos, lo que finalmente impacta la CE (Figura 1).

\section{DISCUSIÓN Y CONCLUSIÓN}

Este estudio buscó determinar las TS que un grupo de padres de una escuela con vulnerabilidad social, tienen acerca de la CE. Se encontraron TS que definen, explican y orientan el rol parental educativo de la CE.

Respecto el significado que los padres le atribuyen a la CE, se observa una TS simple que si bien destaca la necesidad de lograr relaciones interpersonales armónicas en la escuela, estas relaciones sociales solo incluyen a los estudiantes y los profesores, hallazgo que ya se ha advertido en otros trabajos (Saldivia, 2008). No es

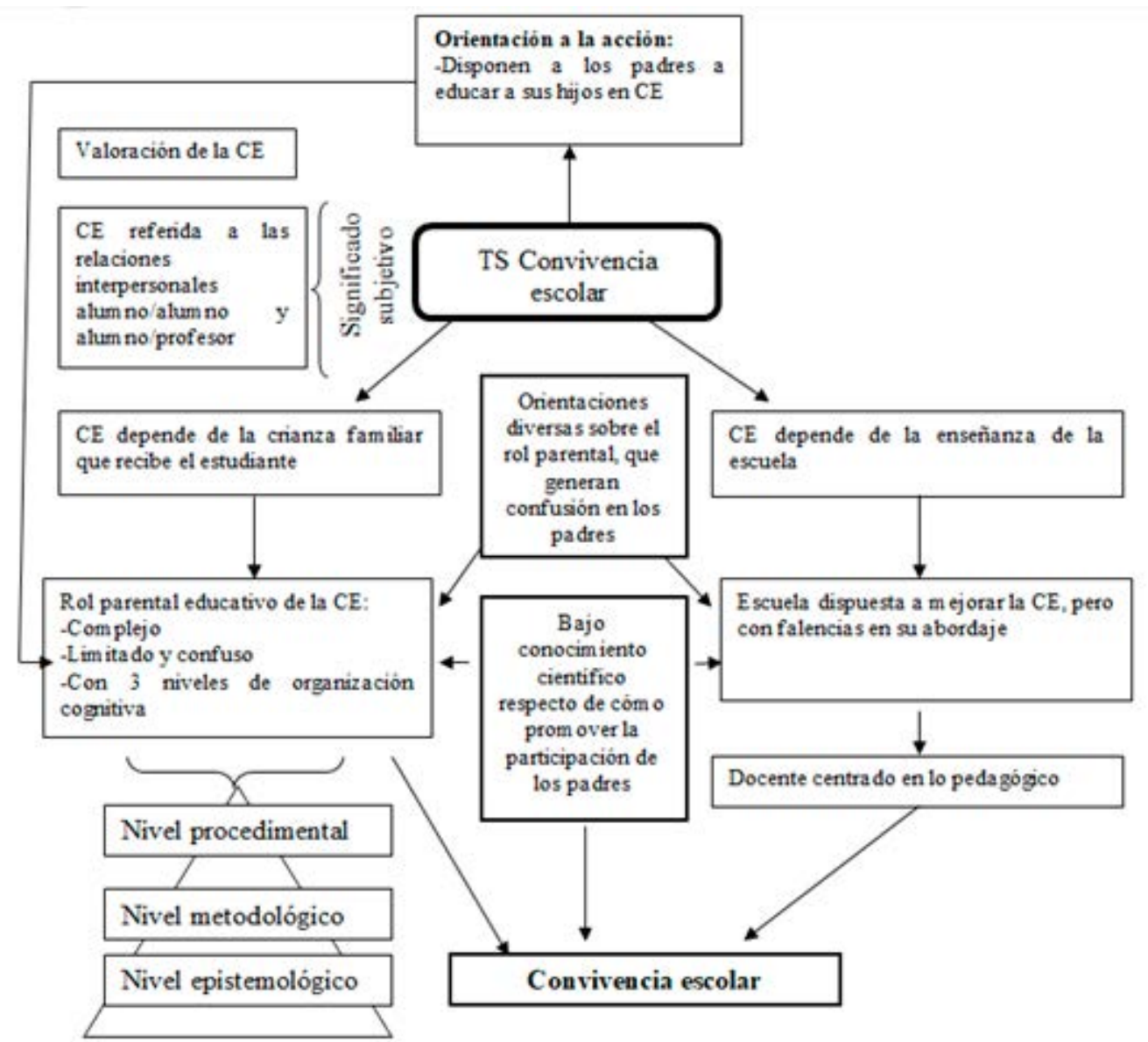

Figura 1. Modelo comprensivo de la CE, desde las TS de los padres. Fuente: elaboración propia. Nota: CE= Convivencia escolar; TS= Teorías sujetivas. 
fácil comprender qué es la CE e incluso en la literatura científica y la política educativa no hay pleno consenso sobre este constructo (Ascorra et al., 2018).

Probablemente la focalización de las escuelas en los contenidos académicos, conlleva a que esta dimensión sea menos razonada, produciéndose múltiples visiones de lo que significa convivir en la escuela, por ejemplo, la naturalización de la indisciplina del estudiante que este grupo de padres considera constituyente de la CE. Esta omisión que hace el apoderado de sí mismo de la red de interacciones escolares, podría asociarse en parte a su crisis participativa en el sistema educativo, sobre todo de aquellas familias con alta vulnerabilidad social (Acuña, 2016; Cid et al., 2008). Desde esto, se desprende la necesidad de generar instancias en donde se discuta y reflexione sobre la $\mathrm{CE}$, incorporando sobre todo las experiencias y la subjetividad de este grupo (por ejemplo, sus TS), dado que el solo acceso al saber subjetivo de las personas desarrolla motivación, involucramiento y dispone al cambio (Helmke, 2009). Es necesario que la comunidad escolar en general comprenda el valor que tiene la CE para el desarrollo del estudiante y de todos los que la integran (Ascorra et al., 2018; Román \& Murillo, 2011; OCDE, 2013).

También se encontraron TS en los padres y madres con atribución interna y externa de la CE. Que en este y otros estudios (López et al., 2011; Saldivia, 2008; Vinha \& Tognetta, 2009), los padres consideren que su rol parental educativo es altamente incidente en el desarrollo de la CE, representa un primer paso en el fortalecimiento de la educación parental. Si bien la literatura internacional muestra que los padres son conscientes de la importancia de su rol en la adaptación escolar y éxito académico de sus hijos(as) (Rahmawati et al., 2018), una dificultad presente es la capacidad que tienen las instituciones educativas para sistematizar un trabajo valorado, razonado, fundamentado y acotado a la realidad educativa, que no solo favorezca una adecuada $C E$, sino que además eduque formalmente a sus alumnos para convivir armónicamente en sociedad.

Respecto de esto último, educar en el rol parental parece no ser una tarea fácil, dado que se presentan contradicciones desde distintas disciplinas y saberes respecto de cómo criar a los hijos (Castro, Van Der Veer, Burgos-Troncoso, Meneses-Pizarro, Pumarino-Cuevas, \& Tello- Viorklumds, 2013; Castro, Cuadra, \& Hu, 2019), situación que sin duda puede conllevar a confusiones en los padres y madres respecto de cómo ejercer su rol parental educativo, en este caso, de la CE. Por nombrar solo un ámbito de contradicción, los cuestionamientos de los padres sobre las leyes de protección a la infancia, como factores que impiden que acciones más rígidas puedan ser utilizadas en la educación de los alumnos, encuentran consonancia con un estudio brasileño en que se identificó la no concordancia tanto de padres y de profesores con la "ley Bernardo", que en Brasil prohíbe que educadores utilicen castigos físicos en la educación de los hijos (Tognetta, 2016). Así como en la investigación brasileña, los padres de este presente estudio parecen considerar el castigo como una herramienta importante para la educación de los hijos.

Un hallazgo novedoso y que requiere de mayor profundización, es la complejidad del aprendizaje de este rol, ya que básicamente incluye tres niveles cognitivos de organización: (a) uno que apela a la naturaleza del conocimiento del rol parental y de cómo los hijos aprenden la $\mathrm{CE}$, que parece representar un cimiento importante para la toma de decisiones acerca de cómo educan y disciplinan a sus hijos; (b) otro metodológico, que apela a los métodos que los padres utilizan para enseñar a sus hijos a convivir en la escuela, siendo predominante en este estudio aquellos centrados en el diálogo y la transmisión de información; y (c) uno práctico procedimental, que implica la enseñanza a sus hijos, de procedimientos conductuales para abordar conflictos interpersonales en la escuela, hallazgo similar al propuesto por Máizquez et al. (2000), al hacer referencia a distintos niveles de actuación en la crianza, incluyendo un nivel estratégico y uno táctico concreto.

Desde lo anterior, la implementación de espacios de fortalecimiento del rol parental debería considerar estos tres niveles cognitivos de organización de la educación parental en CE. Probablemente esto implica abordar un área constituyente de su identidad personal (Fuller, 2000), por lo que se requieren intervenciones que incluyan un cambio subjetivo (Cuadra \& Castro, 2018) y no solo de contenidos y procedimientos.

En las intervenciones psicoeducativas, una forma de considerar la experiencia y saber subjetivo de los padres, es incluyendo las sugerencias que proponen para la mejora de la CE. En este trabajo, proponen el uso de herramientas diversas para mejorar la comunicación con la escuela, por ejemplo, de tecnologías; además de una reorganización de los espacios de encuentro docente - familia. Con respecto al uso de tecnologías de la información y comunicación, Patrikakau (2015) señala que precisamente la participación de los padres en la educación de sus hijos se encuentra en una nueva era, basada en el uso de estas tecnologías, sin embargo, las escuelas parecen ir un paso atrás, resistiéndose a incorporarlas como medio de fomento de la participación.

Ligado a lo anterior, cuando los padres indican acciones protocolares que la escuela puede utilizar para resolver los conflictos en su interior, ofrecen indicativos de acciones que no siempre las escuelas comprenden: los padres entienden la necesidad de que los conflictos 
que ocurren en la escuela sean resueltos al interior de la institución y saben lo que la mayoría de las escuelas aún no han podido comprender: que los conflictos pueden ser oportunidades de aprendizaje de formas más asertivas de resolverlos y así, que es necesario que los profesionales de esta institución no esquiven o "tercericen" los problemas que podrían suceder en este contexto (Vinha \& Tognetta, 2009, 2013).

Finalmente, la participación de la familia en la escuela es una medida clave para el éxito educativo, en este caso para la mejora de la CE. La opción metodológica de este estudio parece pertinente como primer paso para lograr la tan anhelada participación de los padres en la educación de sus hijos, dada la posibilidad de rescatar su subjetividad (las TS), el carácter humanista del paradigma, que favoreces procesos de construcción de realidades, ubicando al participante como protagonista del cambio (Taylor \& Bogdan, 1987), en este caso, a los padres y madres como agentes educativos claves de la CE.

Ciertamente, los países muestran que la participación reducida de los padres en la escuela no es algo que desean. En este caso, los padres también enseñan a las escuelas que más que ser llamados a realizar tareas de apoyo a eventos promovidos por esas instituciones, ellos pueden ser aliados para concretar los mismos objetivos que tienen los profesionales de la educación: llevar a sus hijos y alumnos a la conquista de la ciudadanía.

\section{REFERENCIAS}

Acuña, V. (2016). Familia y escuela: crisis de participación en contextos de vulnerabilidad. Revista Brasileira de Estudos Pedagógicos, 97(246), 255-272. http://dx.doi.org/10.1590/ S2176-6681/267830331

Alcalay, L.; Milicic, N.; Torretti, A. (2005). Alianza efectiva familiaescuela: un programa audiovisual para padres. Psykhe, 14(2), 149-161. doi: 10.4067/S0718-222820050002000295

Ascorra, P.; López, V.; Carrasco, C.; Pizarro, I.; Cuadros, O.; Núñez, C. (2018). Significados atribuidos a la convivencia escolar por equipos directivos, docentes y otros profesionales de escuelas chilenas. Psykhe, 27(1), 1-12.

Castro-Carrasco, P. J.; Cuadra, D.; Hu, C. (2019). Teorías subjetivas sobre disciplina parental en literatura para padres. Rev Chil Pediatr., 90(1), 52-59. doi:10.32641/ rchped.v90i1.811

Castro-carrasco, P. J.; Van der Veer, R.; Burgos-Troncoso, G.; Meneses-Pizarro, L.; Pumarino-Cuevas, N.; TelloViorklumds, C. (2013). Teorías subjetivas en libros latinoamericanos de crianza, acerca de la educación emocional. Revista Latinoamericana de Ciencias Sociales, Niñez y Juventud, 11(2), pp. 703-718.

Catalán, J. (2010). Teorías subjetivas. Aspectos teóricos y prácticos. La Serena: Editorial Universidad de La Serena.
Cid, P.; Díaz, A.; Pérez, M.; Torruella, M.; Valderrama, M. (2008). Agresión y violencia en la escuela como factor de riesgo para el aprendizaje escolar. Ciencia y Enfermería, 14(2), 21-30. http: //www.scielo.cl/pdf/cienf/v14n2/ art04.pdf.

Cuadra, D.; Castro-Carrasco, P. J. (2018). Cambio educativo: propuesta de un modelo de transformación de teorias subjetivas. Liberabit-Revista Peruana de Psicologia, 24(1), 147-164. https://doi.org/https://doi.org/10.24265/ liberabit.2018.v24n1.10

Del Rey, R.; Ortega, R.; Feria, I. (2009). Convivencia escolar: fortaleza de la comunidad educativa y protección ante la conflictividad escolar. Revista Interuniversitaria de Formación del Profesorado, 23(3), 159-180. http://www. aufop.com/aufop/revistas/arta/impresa/139/1273

Flick, U. (2007). Introducción a la investigación cualitativa ( $2^{\text {a }}$ ed.). Madrid: Morata S.L.

Fondo de las Naciones Unidas para la Infancia (2011). Clima, conflictos y violencia en la escuela. Argentina: Facultad Latinoamericana de Ciencias Sociales.

Fuller, N. (2000). Significados y prácticas de paternidad entre varones urbanos del Perú. In Fuller, N. (Ed.), Paternidades en América Latina (pp. 35-89). Lima: Fondo Editorial de la Pontificia Universidad Católica del Perú.

Griffith, J. (2001). Principal leadership of parent involvement. Journal of Educational Administration, (39), 162-186, 2001. http://dx.doi.org/10.1108/09578230110386287.

Helmke, A. (2009). Unterrichtsqualität und lehrerprofessionalität. Diagnose, evaluation und verbesserung des unterrichts. Seelze: Klett Kallmeyer.

Kaufman, S. R. (2009). Transition and school readiness: An outcome of early childhood development. Encyclopedia on early childhood development.

López, C.; Carvajal, C.; Urrea, P.; Soto, M. (2013). Factores asociados a la convivencia escolar en adolescentes. Educación y Educadores, 16(3), 383-410. http:// educacionyeducadores.unisabana.edu.co/index.php/ eye/issue/view/199.

López, V.; Carrasco, C.; Morales, M.; Ayala, A.; López, J.; Karmy, M. (2011). Individualizando la violencia escolar: análisis de prácticas discursivas en una escuela municipal de la región de Valparaíso. Psykhe, 20(2), 7-23. doi: 10.4067/ S0718-22282011000200002.

Máizquez, M.; Rodrigo, M. J.; Capote, C.; Vermaes, I. (2000). Aprender en la vida cotidiana. Un programa experiencial para padres. Madrid: Visor.

Montecinos, C.; Sisto, V.; Ahumada, L. (2010). The construction of parents and teachers as agents for the improvement of municipal schools in Chile. Comparative Education, 46(4), 487-508.

OCDE. (2013). Pesquisa Internacional sobre Ensino $e$ Aprendizagem (TALIS). Recuperado de http://www. oecd.org/edu/school/TALIS-2013-country-note-BrazilPortuguese.pdf 
Patrikakau, E. (2015). Relationship among parents, students, and teachers: The technology wild card. Procedia - Social and Behavioral Sciences, 174(2015), 2253-2258.

Rahmawati, A.; Tairas, M. M.; Nawangsari, N. (2018). Children's school readiness based on teachers' and parents' perceptions. International Journal of Pedagogy and Teacher Education, 2(1), 201-212. doi: 10.20961/ijpte.v\%vi\%i.14876

Román, M.; Murillo, J. (2011). América Latina: violencia entre estudiantes y desempeño escolar. Revista CEPAL, (104), 37-54, 2011. https://www.unicef.org/ecuador/english/ informe-cepal.pdf.

Saldivia, S. (2008). Concepciones asociadas a la convivencia escolar que manejan los docentes directivos, docentes de aula, asistentes de la educación, apoderados y alumnos, que conforman la comunidad educativa de un liceo municipal del sector sur-oriente de la capital. Tesis MA, Universidad de Chile, Santiago.

Sandoval, M. (2014). Convivencia y clima escolar: claves de la gestión del conocimiento. Última Década, 22(41), 153-178, 2014. http://dx.doi.org/10.4067/S071822362014000200007.

Senra, L. X.; Lourenço, L. M.; Pereira, B. O. (2011). Características da relação entre violência doméstica e bullying: revisão sistemática da literatura. Gerais, Revista Interinstitucional de Psicologia, 4(2), 297-309.

Shute, V.; Hansen, E.; Underwood, J.; Razzouk, R.
(2011). A review of the relationship betwen parental involvement and secondary school students' academic achievement. Education Research International. doi: 10.1155/2011/915326

Stake, R. (1999). Investigación con estudio de casos. Madrid: Morata.

Strauss, A.; Corbin, J. (2002). Bases de la investigación cualitativa. Técnicas y procedimientos para desarrollar la teoría fundamentada ( $1^{\circ}$ edición en español). Antioquia: Editorial Universidad de Antioquia.

Taylor, S.; Bogdan, R. (1987). Introducción a los métodos cualitativos de investigación. Barcelona: Paidós.

Tognetta, L. R. P. (2016). O que pensam os pais e professores sobre as formas de educar com castigos e punições? Relatório de pesquisa. FCL/Unesp.

Tognetta, L. R. P.; De Nadai, S. T. (2018). Projeto de formação de pais em escolas brasileiras. Disponível em: http://www. somoscontraobullying.org> Acesso em 25 jul.2018.

Vinha, T. P; Tognetta, L. R. P. (2009). Construindo a autonomia moral na escola: os conflitos interpessoais e a aprendizagem dos valores. Revista Diálogo Educacional, Curitiba, 9(28), 525-540, set./dez.

Vinha, T. P; Tognetta, L. R. P. (2013). A comunicação entre escola e família por meio dos bilhetes ou notificações eletrônicas. In: Anais do III Congresso Internacional de Convivência Escolar. Almeria/Espanha.

Recibido: 18 de marzo de 2019 Aprobado: 19 de septiembre de 2019 